\title{
A Corpus-based Approach to the Quantifiers of Emotion Metaphors in Chinese: A Case Study of Happiness
}

\author{
Wenhui Zhao ${ }^{\text {a }}$, Qin Tang ${ }^{\text {b }}$ \\ Fuyang Normal University, Fu yang, Anhui, China. \\ azwenhui1979@126.com, b1345181630@qq.com
}

Keywords: Emotion metaphor; conceptualization; corpus-based approach; quantifier; happiness.

\begin{abstract}
The major goal of this study is investigate the quantifiers of emotion metaphor "happiness" in Chinese and its English translation, by utilizing a corpus-based analysis. Emotion metaphors are very abstract by nature, there are no quantifiers in English emotion metaphors, but Chinese express their feelings in metaphors with lots of quantifiers. How many kinds of quantifiers are used in Chinese metaphor "happiness"? How to make a vivid English translation? To these end, the current study identify and examines the quantifiers of Chinese metaphorical expression "happiness", and suggest a better English translation.
\end{abstract}

\section{Introduction}

The major goal of this study is to investigate the quantifiers of emotion metaphors of happiness in Chinese by utilizing a corpus-based analysis. The abundant quantifiers are one of the characteristics in modern Chinese language. The existence of rich and unique quantifiers is an important grammatical feature which distinguishes Chinese from English or even Chinese and Tibetan Languages. The quantifiers is a more tangible adjectives and nouns, as well as the grammaticalization of the verbs. The main questions to be answered in this study are (i) how many kinds of quantifiers are used in the metaphorical expressions of HAPPINESS in Chinese? And (ii) how to translate them into English vividly?

The analysis of emotional metaphors in the English language has become a well-developed field of study and continues to expand. In the past two decades, extensive studies have been conducted by cognitive linguists on the function of metaphor and metonymy in the conceptualization of emotion in English (e.g. Kövecses 1990, 1991, 1995a, 1995b, 1999, 2000a, 2000b; Lakoff 1987; Lakoff \& Johnson 1980; Lakoff \& Kövecses 1987). Those studies illustrate how figurative language plays a pivotal role in understanding a phenomenon as complex as the nature of human emotion by analyzing conceptual metaphors or metonymies in the English language. Compared with the studies on English emotion metaphor, there are a few studies of the quantifiers of emotion metaphorical expressions in Chinese (Li 2009:431-438; Yao \&Fan 2002:107-112; Jin\&Chen 2002:8-14; Zing 2001: 68-71). Lakoff and Johnson claim that the most basic metaphors are universal, however they rarely refer to language other than English. More cross-linguistic studies are needed to provide evidence to the theory. Happiness is one of the most basic metaphors in English and Chinese, which are both universal and culture-specific. The quantifiers of emotion metaphor of HAPPINESS in Chinese are culture variations. The choice of these quantifiers is to a certain extent defined by the culture world. In this particular study, I will be providing evidence from Chinese metaphorical expressions of happiness.

This paper proceeds as follows: First, I present a primary case study on emotion lexicons of happiness in Chinese. Second, I provide the main analysis of quantifiers of the metaphorical expressions of happiness in Chinese, applying a corpus-based approach. Third, I suggest a better English translation to these quantifiers. 


\section{Methodology and Scope of the Analysis}

In the case study, I collect the most representative sample of possible happiness-related words based on the Chinese thesaurus "TongYiCi CiLi" [1] by Jiaju Mei(the second edition); and to identify the metaphorical expressions of happiness with quantifiers; and to determine the kinds and amount of the quantifiers; and suggest English translation based on their conceptual meaning.

In the Chinese thesaurus "TongYiCi CiLi", there are 38 lexicons expressing the concept of happiness, which include idioms, adjectives, verbs, single Chinese character and nouns. More metaphorical expressions occur in the noun category than other grammatical categories, thus I choose nouns for the lexical items. For HAPPINESS, idioms are Conventional fixed phrases without any quantifiers to modify, thus I select the two lexical items "xi yue and kuai le". I utilized the corpus of the Centre for Chinese Linguistics PKU (CCL), which includes the corpus of modern Chinese language, the corpus of ancient Chinese language and the corpus of Chinese and English.

The analysis was conducted with the following procedures. First, I collected all the target lexical items used in the corpus, discarding the homonyms. Second, metaphors are the product of conceptual cross-domain mapping, the coded metaphors were examined in terms of context. And then from the metaphorical expressions of HAPPINESS in Chinese, those with quantifiers were further selected. This study provides both qualitative and quantitative information about the quantifiers of HAPPINESS in Chinese.

\section{Corpora Analysis and Translation of Quantifiers in Happiness for Chinese}

Through the corpora analysis, 480 quantifiers [2] and 7 quantifier's patterns were detected for xi yue and kuai le on the concept of HAPPINESS. These quantifiers and quantifiers patterns, listed in table 1, were identified solely based on these two lexical items, which both can be translated as "happy" in English.

In the metaphorical expressions of HAPPINESS in Chinese, the quantifiers are metaphorical by nature. But there is no its own standard to measure the intensity of emotion, thus, others' standards are borrowed to describe the power of emotions, that is mapping.

(1) "yet di" means "a drop of ", which is originally used to measure fluids which is the source domain; the target domain HAPPINESS borrowed "yi di" to express the minimal amount of happiness. The borrowing process, from source domain to target domain, we call mapping. From the table, we can see such a mapping is very rare. When things are "close to zero and more than zero, which are generally weighed by dean". [3] The emotion metaphorical expression in Chinese borrow the concept of "yi dian" to measure the minimal amount or degree, thus the concept of "a little" in English can be matched.

(2) China has a long history of silk, the liking of silk is also reflected in emotion metaphors, "yi si" firstly refers to a silk which is very fine. In emotion metaphors of HAPPINESS, "yi si" is used to describe very small. There is no corresponding quantifier in English, and this quantifier can not be measured accurately. It can only be described in an approximate way according to the degree of emotions. When we make English translation.

(3) Basically, "pain" is a description of "flat and thin" objects. When the concept is mapped into the category of emotions, it means a general description of the shape from a range to a piece, occupying a certain space, so it contains the meaning of "everywhere" and "full". [4]

(4). Read between the lines, "yipping" is used to describe happy people everywhere. "Yet pay" is one synonym of "yipping" in Chinese. It is better to make a free translation: happy people or happy crowds.

(5) "Yi Zhen" represents the duration of a certain situation. The "Zhen" is often used to calculate the actions, things and phenomena that continue for a period of time. "Yet Zhen human lead "yet Zhen xi Yue" means a period of happiness. But the concept of the amount in a Chinese is very weak and obscure, we usually omit such quantifiers in English. 
Table 1. The quantifiers of metaphorical expressions of happiness in Chinese

\begin{tabular}{|c|c|c|}
\hline quantifiers of HAPPINESS in Chinese & $\begin{array}{l}\text { frequency } \\
\text { rating }\end{array}$ & English translation \\
\hline $\begin{array}{c}\text { yi di huan le } \\
\text { yi dian huan le/ xi yue } \\
\text { yi fen xi yue }\end{array}$ & $\begin{array}{c}1 \\
24 \\
1\end{array}$ & a little happiness \\
\hline yi si huan le & 2 & a faint happiness \\
\hline $\begin{array}{l}\text { yipian huan le/ xi yue } \\
\text { yi pai huan le }\end{array}$ & $\begin{array}{c}27 \\
3\end{array}$ & happy people / happy crowds \\
\hline $\begin{array}{l}\text { yi zhen huan le / xi yue } \\
\text { zhen zhen xi yue }\end{array}$ & $\begin{array}{c}26 \\
4\end{array}$ & A period of happiness \\
\hline $\begin{array}{c}\text { yifan/yichang huan le } \\
\text { yi/na gu/fen huan le/ xi yue } \\
\text { yi zhong huan le/ xi yue }\end{array}$ & $\begin{array}{c}2 \\
30 \\
145 \\
\end{array}$ & a happiness/the happiness \\
\hline $\begin{array}{l}\text { yi fen/ ji fen huan le/ xi yue } \\
\text { shi fen huan le/ xi yue }\end{array}$ & $\begin{array}{l}20 \\
38\end{array}$ & $\begin{array}{c}\text { a little happiness } \\
\text { very happy/extremely happy }\end{array}$ \\
\hline $\begin{array}{l}\text { man qiang/ xin/ lian/ huai xi yue } \\
\text { man xin/ huai huan le }\end{array}$ & $\begin{array}{c}155 \\
2\end{array}$ & $\begin{array}{l}\text { full of happiness/ happily/happy } \\
\text { expression }\end{array}$ \\
\hline
\end{tabular}

The concept of "yet si" can be expressed by the adjective word "faint".

(6) The original meaning of "fan" is "animals' footprints in the field" [5]. And "change" refers to "a large place used for a specific purpose" [6]. These two quantifiers modified HAPPINESS mean "number of times". "Yi go" refers to a striped whole. The basic meaning of "yi zhong" is "kind, species or type". "Yi fen" is one part of the whole. These three quantifiers borrowed to signify the quantity of emotion metaphors in Chinese Vaguely. We grammaticalize the concept of these five quantifiers by using the indefinite article "a" or the definite article "the" in English.

(7) Chinese believe that all things can be divided into ten, when Chinese use "yet fen", they mean "a little", "ji fen" has the same concept with that of "little”, the concept of "shí fen" matches "very or extremely" in English.

(8) "Qian, Xin, lain, huai" these quantifiers have a common feature that they are all the external containers for the combination nouns, which we call "Container category quantifiers" [7]. Because of the limitations of sensory, people can't evaluate emotions directly. In order to easily remember and measure the emotions, people often use the external container as a unit to weigh the emotion.

\section{Conclusion}

Quantifiers in emotion metaphors in Chinese describe the abstract feelings indirectly. And there is no necessary, direct similarity between quantifiers and the emotions modified. The quantifiers in the emotion metaphorical expressions in Chinese can describe people's emotions accurately and vividly, because they can measure the intangible emotions by using the tangible things with stereoscopic and concrete images.

\section{Acknowledgments}

This work was partially supported by grants from the Foundations of Department of Education in Anhui province (gxyqZD2017090, AHSKQ2016D120, SK2015A720, 2016jyxm0751, 2017FSKJ14,) to Fuyang Normal University.

\section{References}

[1]. Juju Mei et al. 1996, TongYiCi Cilia, second ed., Shanghai: Shanghai Lexicographic Press, pp. 229. 
[2]. The corpus of the Centre for Chinese Linguistics PKU: http://ccl.pku.edu.cn/corpus.asp (last checked on Dec. 5th 2017).

[3]. Weismann. 2011, Cognitive linguistics and the study of Chinese, first ed., Shanghai: Fudan university Press.

[4]. Yan, L. 1999, “On the Collocation of Quantifiers and Adjectives in Chinese” J. Chinese Language Learning, (5): 60-63.

[5]. Badu: https://baike.baidu.com/item/4573828 (last checked on Dec. 5th 2017).

[6]. Badu: https://baike.baidu.com/item//69842 (last checked on Dec. 5th 2017).

[7]. Zhen bang, D., Young, L. 2015, “On the Chinese Quantifiers” J. Academic Exploration, (9): 134-137. 